\title{
Dynamical analysis of a fractional SIRS model on homogenous networks
}

\author{
H.A.A. El-Saka ${ }^{1 *}$, A.A.M. Arafa ${ }^{2}$ and M.I. Gouda ${ }^{2}$
}

"Correspondence: halaelsaka@yahoo.com

${ }^{1}$ Mathematics Department, Faculty of Science, Damietta University, New Damietta, Egypt Full list of author information is available at the end of the article

\begin{abstract}
In this paper, we propose a fractional SIRS model with homogenous networks. The disease-free equilibrium point $E_{0}$ is locally and globally asymptotically stable for $R_{0}<1$ (the disease always disappears), and endemic equilibrium point $E_{1}$ is uniquely locally and globally asymptotically stable, but $E_{0}$ is unstable for $R_{0}>1$ (the disease is uniformly persistent). The main results are demonstrated by numerical simulation.
\end{abstract}

Keywords: Fractional order SIRS model; Homogenous network; Local stability; Global stability; Numerical solutions

\section{Introduction}

Spreading of infections has been studied by researchers who used mathematical modeling based on complex networks, which help us to understand the infections, to predict the effect of those infections on our population, to prevent and control most dangerous infectious diseases like HIV/AIDS, dengue fever, and H1N1 [1-3], and decide whether they are epidemic or nonepidemic. Complex networks consist of nodes and links that connect the nodes. We can represent each individual of the population, which is divided into three categories (Susceptible " $x$ ", infected " $y$ ", and removed " $z$ ") as nodes, and represent links as social communication. The epidemic spreading process based on homogenous networks is discussed in [4], and we can note that the main factor in homogenous network is the average degree of nodes, which is the average contact between individuals in the population.

In [5] the modified SIRS epidemic model is introduced, and the count in a nonlinear term plays an important role in the calculation of reproductive ratio $R_{0}$, which means that the connection between individuals helps us to decide whether the disease is epidemic or free in the population. In this paper, we introduce the SIRS model supported by fractional differential equations.

It is commonly known that fractional-order systems are an extension of conventional integer-order systems. However, it should be noted that they possess advantages over the conventional integer-order systems, on which we base our work. Those advantages include having memory and hereditary properties that integer-order systems do not, which are usually implemented by most biological systems. Another advantage is that fractionalorder systems have a more accurate description of population models than that of the integer-order ones; they also better indicate relations between prey and predator species. 
Needless to mention a wide spread of applications of fractional-order derivatives to interdisciplinary fields. When it comes to memory and hereditary properties of materials or processes, it is often well pointed out that fractional-order calculus is best suited to their description. Such properties are all but neglected by the classical integer-order models. Considering many intriguing results found while working on models of the dynamics of population, they were found to be exclusive to integer-order differential equations [6,7].

Mainly, a great recognition and credit earned by fractional-order differential equations, as of late, has been based on the natural compatibility and better relation to systems with memory, which is found in most biological systems. Not failing to mention their relation to fractals, fractional-order systems are doubtlessly better related to them than integerorder systems, and fractals being quite abundant in biological systems, it is needless to further question the suitability of fractional-order systems to our work. Having recognized the need to use fractional-order systems, many biological phenomena and interdisciplinary fields have been precisely and successfully described through models using fractional-order systems. Due to the scarcity of theories needed to analyze the dynamics of fractional-order systems, studies of stability of fractional-order population models can only be the beginning of a vast and doubtlessly fruitful implementation [6, 7]. This study is also motivated by Zika epidemic [8].

In the rest of the paper, we discuss the effect of fractional differential equations and the role of networks, especially in local and global stability, and we finally support our work by numerical results. Some integral and derivative basics of the fractional order and some preliminary results are mentioned in Sect. 2. In Sect. 3, we give the model. In Sect. 4, equilibria are calculated with studies on local stability. The global stability of equilibria is studied in Sect. 5. In Sect. 6, we use a numerical method (Adams-type predictor-corrector method) to solve our model in order to support the theoretical results. Finally, conclusions are summarized in Sect. 7.

\section{Preliminaries}

First, we illustrate the definitions of the fractional-order integral, Caputo fractional derivative, and Mittag-Leffler function; see [9].

Definition 2.1 The fractional integral of order $q \in R^{+}$of the function $g(t), t>0$, is defined by

$$
I^{q} g(t)=\int_{0}^{t} \frac{(t-s)^{q-1}}{\Gamma(q)} g(s) d s
$$

Definition 2.2 The Caputo fractional derivative of order $q>0$ of $g(t), t>0$, is defined by

$$
D_{*}^{q} g(t)=I^{n-q} D^{n} g(t)
$$

where $D=d / d t$ and $n-1<q \leq n, n \in \mathbb{N}$.

For properties of fractional derivatives and integrals, see [9]. 
Definition 2.3 The Mittag-Leffler function of parameter $q>0$ is defined as

$$
E_{q}(z)=\sum_{j=0}^{\infty} \frac{z^{j}}{\Gamma(q j+1)}
$$

Let $q \in(0,1]$ and consider the system

$$
\begin{aligned}
& D_{*}^{q} y_{1}(t)=g_{1}\left(y_{1}, y_{2}, y_{3}\right), \\
& D_{*}^{q} y_{2}(t)=g_{2}\left(y_{1}, y_{2}, y_{3}\right), \\
& D_{*}^{q} y_{3}(t)=g_{3}\left(y_{1}, y_{2}, y_{3}\right), \\
& y_{1}(0)=y_{o 1}, \quad y_{2}(0)=y_{o 2}, \quad \text { and } y_{3}(0)=y_{o 3} .
\end{aligned}
$$

Definition 2.4 The constant $\left(y_{1}^{\mathrm{eq}}, y_{2}^{\mathrm{eq}}, y_{3}^{\mathrm{eq}}\right)$ can only be an equilibrium point of the fractional dynamic model (4) if and only if

$$
g_{i}\left(y_{1}^{\mathrm{eq}}, y_{2}^{\mathrm{eq}}, y_{3}^{\mathrm{eq}}\right)=0, \quad i=1,2,3
$$

Theorem 2.1 The equilibrium points of system (4) are locally asymptotically stable (LAS) if all eigenvalues $r_{i}$ of the Jacobian matrix evaluated at the equilibrium points

$$
\begin{aligned}
& B=\left[\begin{array}{lll}
b_{11} & b_{12} & b_{13} \\
b_{21} & b_{22} & b_{23} \\
b_{31} & b_{32} & b_{33}
\end{array}\right], \\
& b_{i j}=\left.\frac{\partial g_{i}}{\partial y_{j}}\right|_{\mathrm{eq}}, \quad i, j=1,2,3,
\end{aligned}
$$

satisfy

$$
\left|\arg \left(r_{i}\right)\right|>\frac{q \pi}{2}, \quad i=1,2,3
$$

Proof Refer to [10-13].

Figure 1 shows the condition of the stability of the fractional-order model with order $\alpha$. We use the following polynomial to obtain an equation for the eigenvalues of the equilibrium point $\left(y_{1}^{\mathrm{eq}}, y_{2}^{\mathrm{eq}}, y_{3}^{\mathrm{eq}}\right)$ :

$$
p(r)=r^{3}+b_{1} r^{2}+b_{2} r+b_{3}=0
$$

and its discriminant $D(P)$ is given by

$$
D(P)=-\left|\begin{array}{ccccc}
1 & b_{1} & b_{2} & b_{3} & 0 \\
0 & 1 & b_{1} & b_{2} & b_{3} \\
3 & 2 b_{1} & b_{2} & 0 & 0 \\
0 & 3 & 2 b_{1} & b_{2} & 0 \\
0 & 0 & 3 & 2 b_{1} & b_{2}
\end{array}\right|=18 b_{1} b_{2} b_{3}+\left(b_{1} b_{2}\right)^{2}-4 b_{3} b_{1}^{3}-4 b_{2}^{3}-27 b_{3}^{2}
$$


Figure 1 Stability region of the fractional-order system

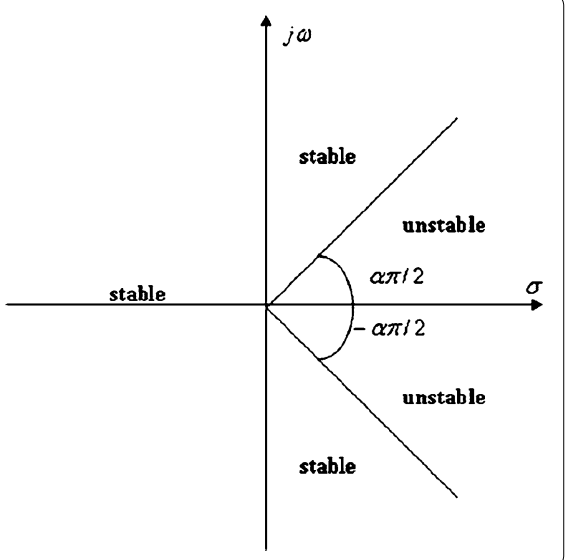

Proposition 2.1 ([10]) The equilibrium point $\left(y_{1}^{\mathrm{eq}}, y_{2}^{\mathrm{eq}}, y_{3}^{\mathrm{eq}}\right)$ of system (4) is locally asymptotically stable if one of the following conditions is satisfied:

(i) $D(P)>0, b_{1}>0, b_{3}>0$, and $b_{1} b_{2}-b_{3}>0$.

(ii) $D(P)<0, b_{1} \geq 0, b_{2} \geq 0, b_{3}>0$, and $q<2 / 3$.

(iii) $D(P)<0, b_{1}>0, b_{2}>0, b_{1} b_{2}-b_{3}=0$, and for all $q \in(0,1)$.

(iv) The imperative condition is $b_{3}>0$.

Now, consider the following autonomous system:

$$
D_{*}^{q} y(t)=g(y), \quad q \in(0,1)
$$

The following lemmas help us to prove the globally asymptotical stability (GAS) of equilibrium points.

Lemma 2.2 (See [14]) Suppose D is a bounded closed set. Every solution of (9) starts from a point in $D$ and remains in $D$ all the time. If $\exists V(y): D \longrightarrow \mathbb{R}$ with continuous first partial derivatives satisfies following condition:

$$
\left.D_{*}^{q} V\right|_{(9)} \leq 0 \text {. }
$$

Let $E=\left\{\left.D_{*}^{q} V\right|_{(9)}=0, y \in D\right\}$, and let $M$ be the largest invariant set of $E$. Then every solution $y(t)$ originating in $D$ tends to $M$ as $t \rightarrow \infty$. Particularly, if $M=\{0\}$, then $y \rightarrow 0$ as $t \rightarrow \infty$.

Lemma 2.3 (See [15]) Let $y(t) \in \mathbb{R}^{+}$be a continuous and differentiable function. Then, for any time instant $t \geq t_{0}$,

$$
D_{*}^{q}\left[y(t)-y^{*}-y^{*} \ln \frac{y(t)}{y^{*}}\right] \leq\left(1-\frac{y^{*}}{y(t)}\right) D_{*}^{q} y(t), \quad y^{*} \in \mathbb{R}^{+}, \forall q \in(0,1) .
$$

\section{SIRS epidemic model on homogenous networks with fractional order (FSIRS)}

Let $x(t)$ be the number of susceptible individuals, let $y(t)$ represent the infected individuals, and let $z(t)$ represent the recovered or vaccinated individuals; all individuals are considered at time $t[5]$. 


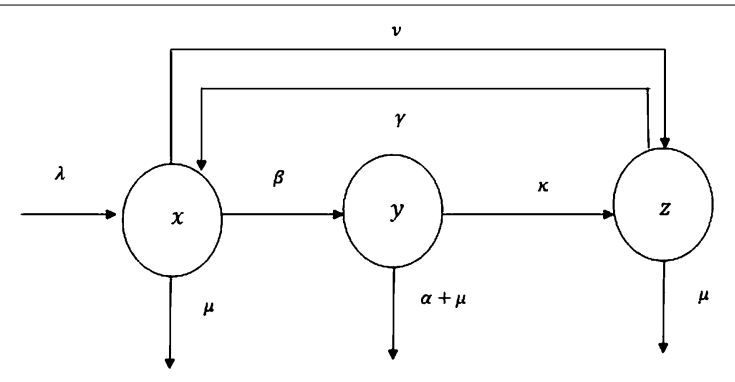

Figure 2 Flowchart of transmission of disease

The FSIRS model of homogenous networks is given by

$$
\begin{aligned}
& D_{*}^{q} x(t)=\lambda-\frac{\beta\langle k\rangle x y}{x+y+z}+\gamma z-(v+\mu) x, \\
& D_{*}^{q} y(t)=\frac{\beta\langle k\rangle x y}{x+y+z}-(\kappa+\mu+\alpha) y, \\
& D_{*}^{q} z(t)=\kappa y-(\mu+\gamma) z+v x,
\end{aligned}
$$

where $D_{*}^{q}$ is the Caputo derivative, $0<q \leq 1$, and all the parameters $\mu, \kappa, \lambda, \beta, v, \gamma$, and $\alpha$ are positive.

The parameter $\beta$ represent the infection rate (the rate of susceptible individuals infected due to contact with infected individuals), $\kappa$ is the healing rate, and healed individuals become susceptible at the rate $\gamma$. At the same time, $v$ represents the inoculation rate (susceptible individuals acquiring immunity by drug). The constant $\lambda$ is the change rate of susceptibles due to birth and immigration, where $\mu$ is the death rate of population independently of disease, and $\alpha$ is the death rate due to disease. Figure 2 describes the transmission of disease.

Given that the network is homogenous, we assume that $\langle k\rangle$ is the average connection between nodes in the network and that all nodes have unique degrees. Also, the infection has a positive relation with population density [5].

We denote the total population as $N=x+y+z$.

Theorem 3.1 The closed set $\Omega=\left\{(x, y, z) \in \mathbb{R}_{+}^{3}: x \geq 0, y \geq 0, z \geq 0, x+y+z \leq \frac{\lambda}{\mu}\right\}$ is a positive invariant set and global attracting set of system (11).

Proof Adding three classes of system (11), we get:

$$
\begin{aligned}
& D_{*}^{q} N=\lambda-\mu N-\alpha y, \\
& D_{*}^{q} N \leq \lambda-\mu N .
\end{aligned}
$$

Applying the Laplace transform, we obtain:

$$
N(t) \leq\left(-\frac{\lambda}{\mu}+N(0)\right) E_{q}\left(-\mu t^{q}\right)+\frac{\lambda}{\mu},
$$

where $E_{q}\left(-\mu t^{q}\right)$ is the Mittag-Leffer function of parameter $q$. 
Especially, if $N(0) \leq \frac{\lambda}{\mu}$, then $N(t) \leq \frac{\lambda}{\mu}$, since $0 \leq E_{q}\left(-\mu t^{q}\right) \leq 1$, so the closed set $\Omega$ is the positive invariant set of system (11). Furthermore, since $\lim _{t \rightarrow \infty} E_{q}\left(-\mu t^{q}\right)=0$, it follows that if $N(0)>\frac{\lambda}{\mu}$, then the solution $\phi(N(t))$ of system (11) satisfies $\lim _{t \rightarrow \infty} \phi(N(t))=\frac{\lambda}{\mu}$. Hence the closed set $\Omega$ attracts all the solutions in $\mathbb{R}_{+}^{3}$, so that $\Omega$ is the global attracting set of system (11).

\section{Equilibria and their local stability}

The equilibrium points of system $(11)$ are $\left(x_{0}, y_{0}, z_{0}\right)=\left(\frac{\lambda(\gamma+\mu)}{\mu(\gamma+\mu+v)}, 0, \frac{\lambda v}{\mu(\gamma+\mu+v)}\right)$ and $\left(x^{*}, y^{*}, z^{*}\right)$, where

$$
\begin{aligned}
x^{*} & =\frac{\lambda \rho(\kappa+\gamma+\mu)}{\left.\rho \zeta\left[\mu R_{0}+\alpha\left(R_{0}-1\right)\right]+\beta\langle k\rangle \mu \kappa\right]}, \\
y^{*} & =\frac{\lambda \rho \zeta\left(R_{0}-1\right)}{\left.\rho \zeta\left[\mu R_{0}+\alpha\left(R_{0}-1\right)\right]+\beta\langle k\rangle \mu \kappa\right]}, \\
z^{*} & =\frac{\kappa y^{*}+v x^{*}}{\mu+\gamma}, \\
R_{0} & =\frac{\beta\langle k\rangle(\mu+\gamma)}{(\kappa+\alpha+\mu)(\mu+v+\gamma)}, \\
\rho & =(\kappa+\alpha+\mu), \\
\zeta & =(\mu+v+\gamma) .
\end{aligned}
$$

\section{Theorem 4.1}

(i) System (11) has a unique disease-free equilibrium $E_{0}=\left(\frac{\lambda(\gamma+\mu)}{\mu(\gamma+\mu+v)}, 0, \frac{\lambda v}{\mu(\gamma+\mu+v)}\right)$ under the condition $R_{0} \leq 1$.

(ii) If $R_{0}>1$, then $E_{0}$ still exists, and system (11) has a unique endemic equilibrium $E_{1}=\left(x^{*}, y^{*}, z^{*}\right)$.

The local stability of $E_{0}$ and $E_{1}$ is studied in the following theorems.

Theorem 4.2 The disease-free equilibrium $E_{0}$ is locally asymptotically stable if $R_{0}<1$ and is unstable whenever $R_{0}>1$.

Proof Using the Jacobian matrix of system (11) at $E_{0}$, we find that the eigenvalues are:

$$
\begin{aligned}
& r_{1}=-\mu<0, \\
& r_{2}=-(\mu+\gamma+v)<0, \\
& r_{3}=-(\kappa+\mu+\alpha)\left(1-R_{0}\right)<0 .
\end{aligned}
$$

Hence $E_{0}$ is locally asymptotically stable if $R_{0}<1$.

Now, to study the local stability of $E_{1}$, we assume that $R_{0}>1$. After evaluating the Jacobian matrix of system (11) at $E_{1}$, the characteristic polynomial is given by

$$
r^{3}+a_{1} r^{2}+a_{2} r+a_{3}=0
$$


where

$$
\begin{aligned}
& a_{1}=\gamma+2 \mu+v+p w, \\
& a_{2}=(p-\tau) w^{2}+(\gamma p+\kappa \tau+p \mu+\tau \mu) w+\gamma \mu+\mu v+\mu^{2}, \\
& a_{3}=[p(\gamma+\mu)-\tau(\mu+v+\gamma)] w^{2}+[\tau(\mu+v+\gamma)(\mu+\kappa)-\gamma \kappa p] w, \\
& p=\frac{y^{*}}{x^{*}}, \quad \tau=\frac{y^{*}}{N^{*}}, \quad N^{*}=x^{*}+y^{*}+z^{*}, \quad(p-\tau)>0, \\
& w=(\alpha+\mu+\kappa)=\frac{\beta\langle k\rangle x^{*}}{N^{*}} .
\end{aligned}
$$

It is obvious that $a_{1}, a_{2}>0$ and $a_{3}=\tau(\mu+v+\gamma)\left[\alpha\left(R_{0}-1\right)+\mu R_{0}+\frac{\mu \kappa\langle k\rangle \beta}{(\alpha+\mu+\kappa)(\mu+v+\gamma)}\right]>0$ as long as $R_{0}>1$. Further, we have $a_{1} a_{2}-a_{3}>0$.

So, the Routh-Hurwitz conditions are satisfied. Let $D(p)$ denote the discriminant of polynomial (12) given by (8); using Proposition 2.1, we obtain the following result.

Theorem 4.3 Assume that $R_{0}>1$.

(i) If $D(p)>0$, then $E_{1}$ is locally asymptotically stable for all $0<q \leq 1$.

(ii) If $D(p)<0$ and $q<2 / 3$, then $E_{1}$ is locally asymptotically stable.

\section{Global stability}

The global stability of $E_{0}$ and $E_{1}$ are studied by using a Lyapunov function.

Theorem 5.1 If $R_{0}<1$, then the disease-free equilibrium $E_{0}$ is globally asymptotically stable.

Proof For system (11), consider the following Lyapunov function:

$$
L_{0}(t)=y \text {. }
$$

On calculating $D_{*}^{q} L_{0}$, we get:

$$
\begin{aligned}
& D_{*}^{q} L_{0}=y\left(\frac{\beta\langle k\rangle x}{N}-\rho\right), \\
& D_{*}^{q} L_{0} \leq \rho y\left(\frac{\beta\langle k\rangle(\gamma+\mu)}{\rho(\gamma+\mu+v)}-1\right), \\
& D_{*}^{q} L_{0} \leq \rho y\left(R_{0}-1\right) .
\end{aligned}
$$

Then

$$
D_{*}^{q} L_{0} \leq 0 \quad \text { if } R_{0}<1 .
$$

Hence, if $R_{0}<1$, then $D_{*}^{q} L_{0} \leq 0$. Furthermore, the largest invariant set of $\left\{(x, y, z): D_{*}^{q} L_{0}=\right.$ $0\}$ is the singleton $\left\{E_{0}\right\}$. According to Lemma 2.2 and Theorem 4.2, $E_{0}$ is globally asymptotically stable when $R_{0}<1$, which implies that the disease will disappear regardless of the initial density of the infected individuals. 
Theorem 5.2 If $R_{0}>1$, then $E_{1}$ is globally asymptotically stable.

Proof Define the Lyapunov functional $L_{1}(t)$ as follows:

$$
L_{1}(t)=x^{*} \Phi\left(\frac{x}{x^{*}}\right)+y^{*} \Phi\left(\frac{y}{y^{*}}\right)+z^{*} \Phi\left(\frac{z}{z^{*}}\right)
$$

where $\Phi(y)=y-1-\ln (y), y>0$. It is obvious that $\Phi(y)>0$.

Calculating $D_{*}^{q} L_{1}$ and using Lemma 2.3, we get:

$$
D_{*}^{q} L_{1} \leq\left(\frac{x-x^{*}}{x}\right) D_{*}^{q} x+\left(\frac{y-y^{*}}{y}\right) D_{*}^{q} y+\left(\frac{z-z^{*}}{z}\right) D_{*}^{q} z,
$$

where $\lambda=\frac{\beta\langle k\rangle x^{*} y^{*}}{N^{*}}-\gamma z^{*}+(v+\mu) x^{*}$. Hence

$$
\begin{aligned}
D_{*}^{q} L_{1} \leq & -(v+\mu) \frac{\left(x-x^{*}\right)^{2}}{x}-\frac{\beta\langle k\rangle y^{*}}{x N^{*}}\left(x-x^{*}\right)^{2} \\
& +\frac{\beta\langle k\rangle\left(N-N^{*}\right)}{N N^{*}}\left(x y^{*}-x^{*} y\right) \\
& -\left(z-z^{*}\right)(\mu+\gamma)+\left(\frac{z-z^{*}}{z}\right)(\kappa y+v x)+\gamma\left(z-z^{*}\right)\left(\frac{x-x^{*}}{x}\right) \\
\leq & -(v+\mu) \frac{\left(x-x^{*}\right)^{2}}{x}-\frac{\beta\langle k\rangle y^{*}}{x N^{*}}\left(x-x^{*}\right)^{2}-\frac{\beta\langle k\rangle x y^{*}}{N^{*}} \Phi\left(\frac{N^{*}}{N}\right) \\
& -\frac{\beta\langle k\rangle x^{*} y}{N} \Phi\left(\frac{N}{N^{*}}\right)-(\kappa y+v x) \Phi\left(\frac{z^{*}}{z}\right)-z^{*}\left(\mu+\gamma \frac{x^{*}}{x}\right) \Phi\left(\frac{z}{z^{*}}\right) .
\end{aligned}
$$

Hence $D_{*}^{q} L_{1} \leq 0$. Furthermore, the largest invariant set of $\left\{(x, y, z): D_{*}^{q} L_{1}=0\right\}$ is the singleton $\left\{E_{1}\right\}$. According to Lemma 2.2 and Theorem 4.2, $E_{1}$ is globally asymptotically stable, which implies that the disease is still at an endemic level, and it does not depend on the initial density of the infected individuals. This result leads us to wonder how we can reduce the basic reproduction number $R_{0}$ to be less than one.

\section{Numerical methods and results}

The original problem (11) is equivalent to the following fractional integral equations:

$$
\begin{aligned}
& x(t)=x(0)+I^{q}\left[\lambda-\frac{\beta\langle k\rangle x y}{x+y+z}+\gamma z-(v+\mu) x\right], \\
& y(t)=y(0)+I^{q}\left[\frac{\beta\langle k\rangle x y}{x+y+z}-(\kappa+\mu+\alpha) y\right], \\
& z(t)=z(0)+I^{q}[\kappa y-(\mu+\gamma) z+v x] .
\end{aligned}
$$

An Adams-type predictor-corrector method [16-18] is used for the numerical solution of fractional integral equations.

$x(t), y(t)$, and $z(t)$ are displayed in Figs. 3-8. In each figure, three different values of $q=1,0.95,0.90$, as well as the parameters, are considered as in Table 1. In Figs. 3-5, 

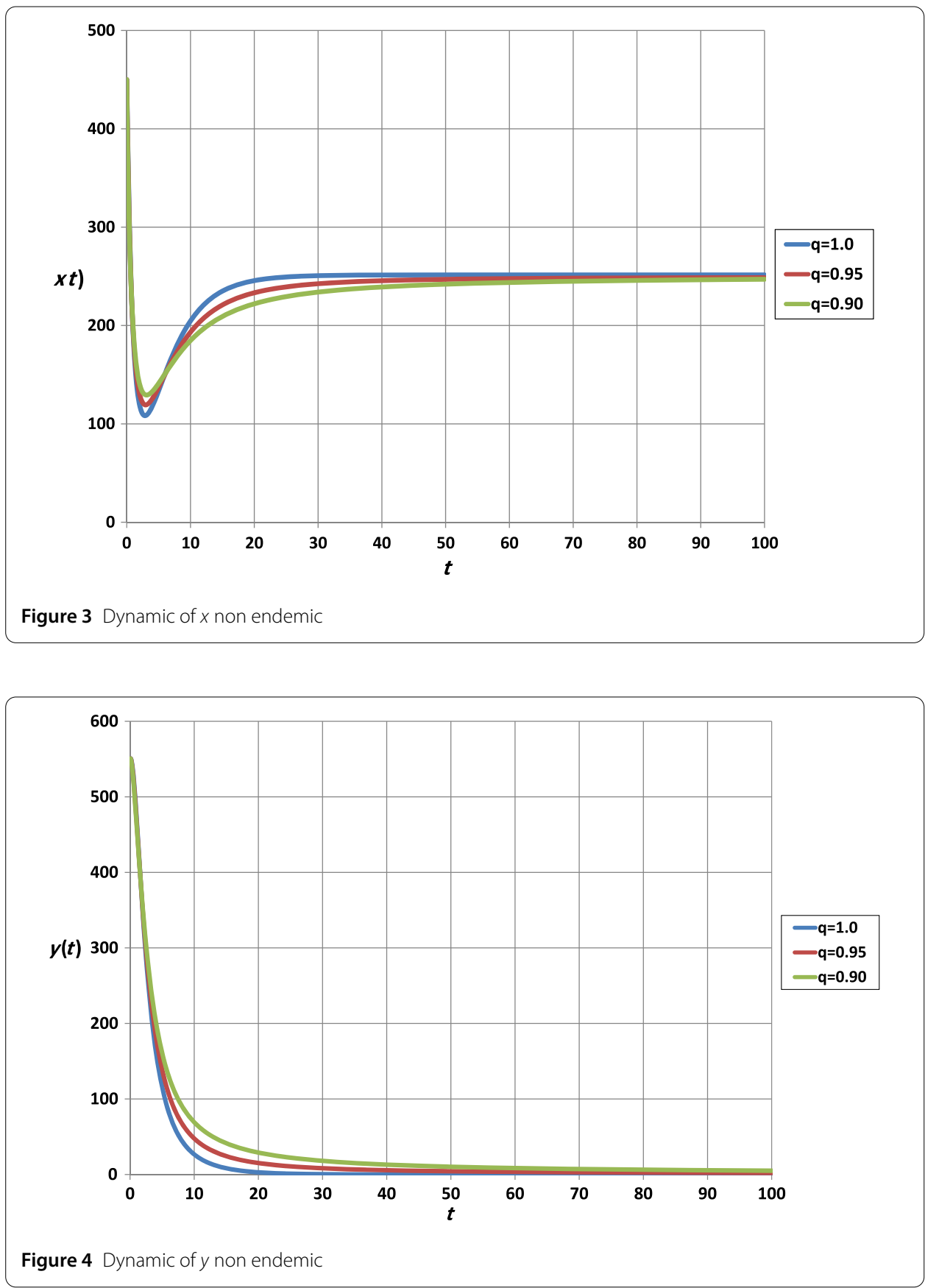

we set $v=0.3$, and the initial conditions are $(450,550,0)$. In this case, the value of $R_{0}$ is $R_{0}=0.602236<1$, which means that $E_{0}=(251.87,0,748.13)$ is globally asymptotically stable, albeit for a large number of infected individuals at the initial time, which means the infection will die out in the population.

In Figs. $6-8$, we set $v=0.005$, and the initial conditions are $(800,200,0)$. In this case, the value of $R_{0}$ is $R_{0}=2.27827>1$, which means that $E_{0}=(952.83,0,47.1698)$ is unstable, and a unique endemic equilibrium point $E_{1}=(386.518,87.1414,450.528)$ is globally asymptotically stable, albeit for a small number of infected individuals at the initial time, which means that the infection will persist in the population. 

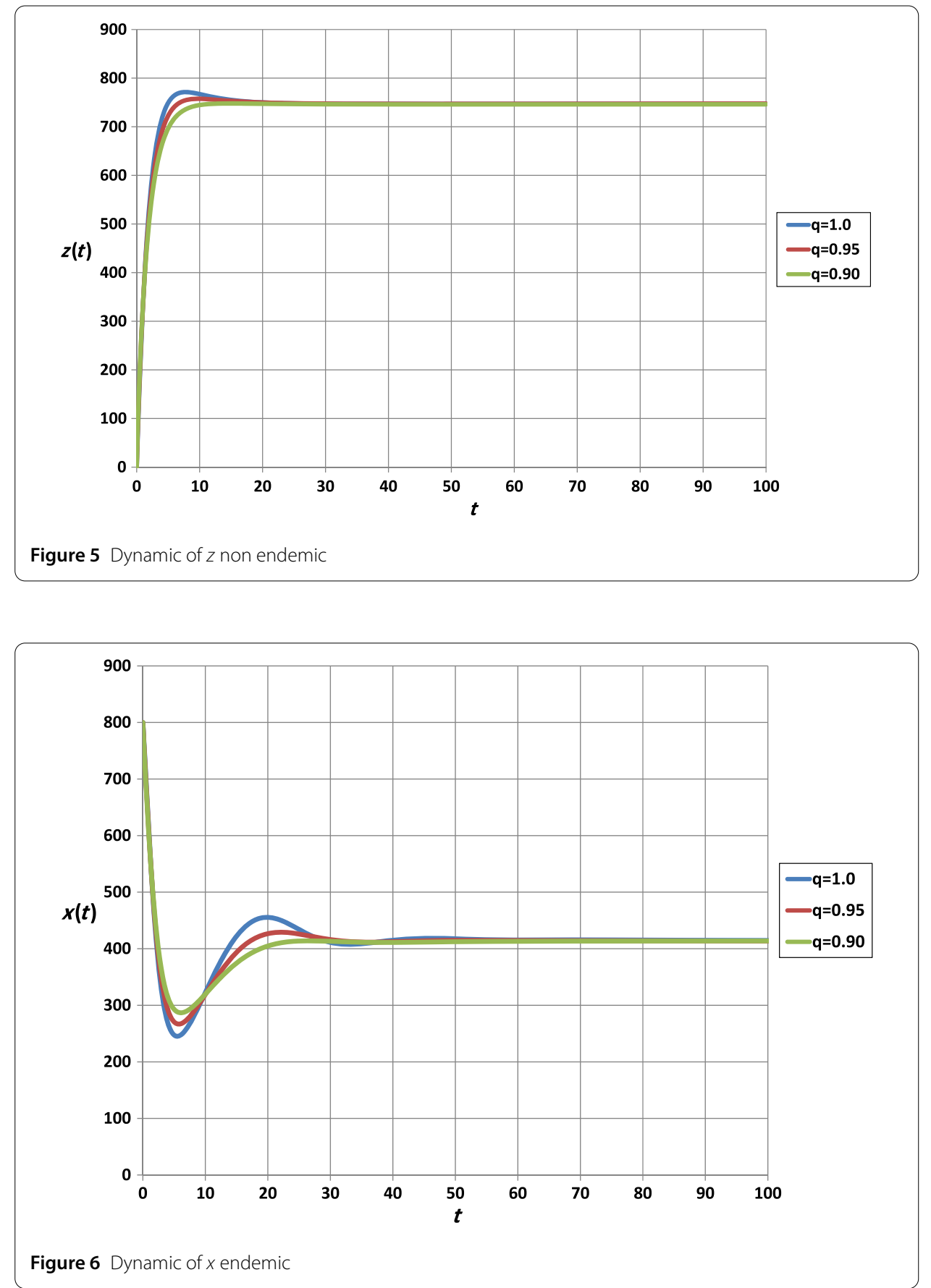

Now, we study Hepatitis B virus infection in China with clinal data [5] for the parameter values of system (11) as in Table 2 , with the initial conditions $\left(4.0 \times 10^{8}, 8.56 \times 10^{7}, 6.54 \times\right.$ $\left.10^{8}\right)$.

The approximate solutions $y(t)$ are displayed in Figs. 9-11 for $q=1,0.99,0.98$ and $v=$ $0.2707,0.5,0.9$, and we find that $R_{0}<1$.

For $v=0.2707$, the data show that the estimate of the infected individuals in 2010 is about 120361000 for $q=1,120115900$ for $q=0.99$ and 119871900 for $q=0.98$, which is close to the real estimate of HBV-infected individuals 120 million in China in 2010 [19]. 

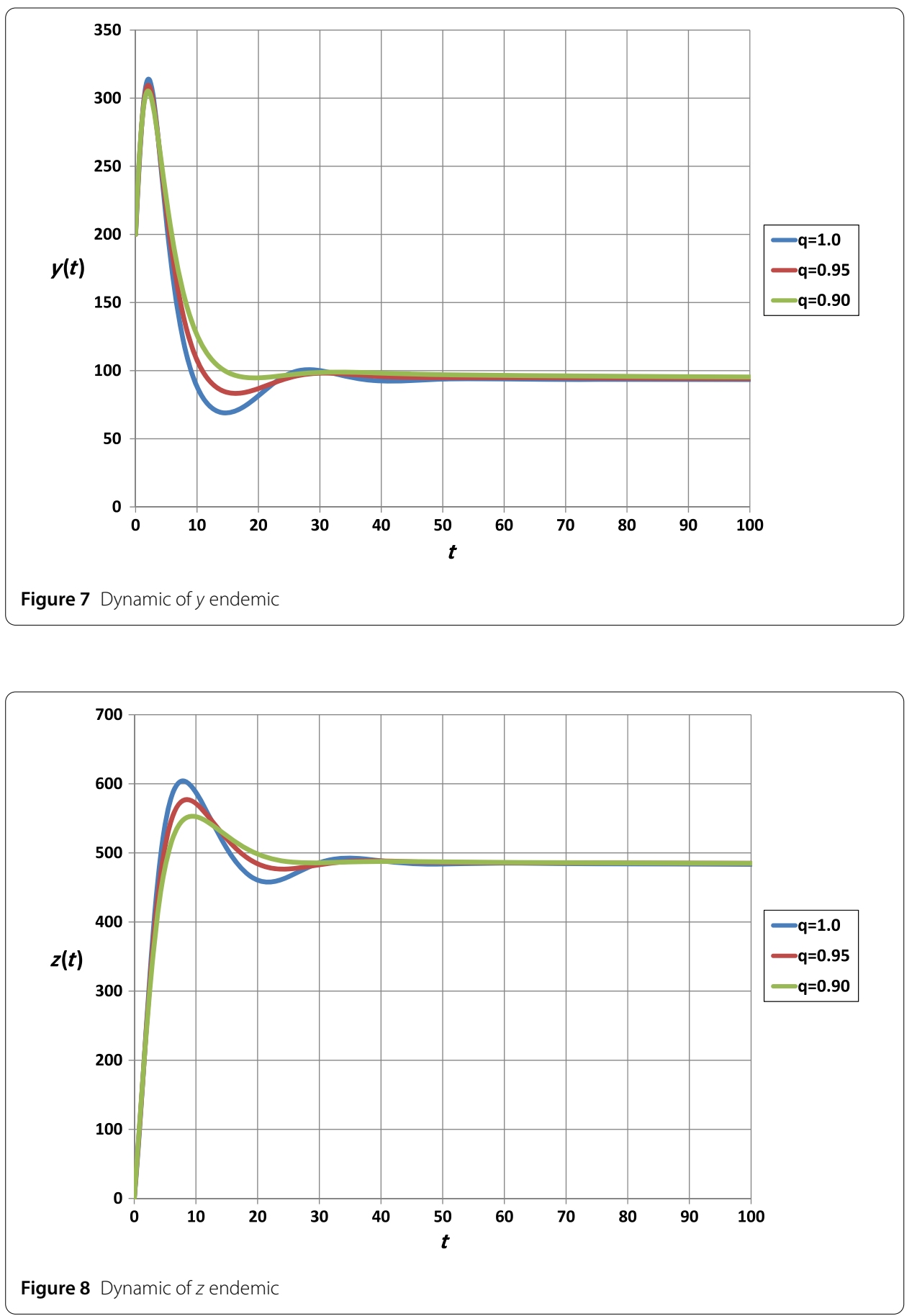

Over a long period of time, numerical results predict that the ratio of the HBV-infected individuals will decrease to $0.5 \%$ passing by different years for $q=1,0.99,0.98$ as in Table 3 .

The level represented is the same level of the HBV-infected population ratio in Northern America [20]. The relation between the vaccinated population and time is shown in Tables 4 and 5 (if the percentage of $(v)$ of the immunized population changes to $v=0.5$ and $v=0.9$, then the required time to reduce to $0.5 \% \mathrm{HBV}$-infected population will decrease). 
Table 1 Parameter values for simulations

\begin{tabular}{ll}
\hline Parameter & Value \\
\hline$\langle k\rangle$ & 6 \\
$\kappa$ & 0.5 \\
$\alpha$ & 0.00087 \\
$\gamma$ & 0.1 \\
$\mu$ & 0.001 \\
$\beta$ & 0.2 \\
$\lambda$ & 1 \\
\hline
\end{tabular}

Table 2 Parameter values for simulations

\begin{tabular}{ll}
\hline Parameter & Value \\
\hline$\langle k\rangle$ & 6 \\
$\kappa$ & 0.073 \\
$\mu$ & 0.00714 \\
$\beta$ & 0.13 \\
$\alpha$ & 0.0007 \\
$\gamma$ & 0.01 \\
$\lambda$ & $13,552,480$ \\
\hline
\end{tabular}

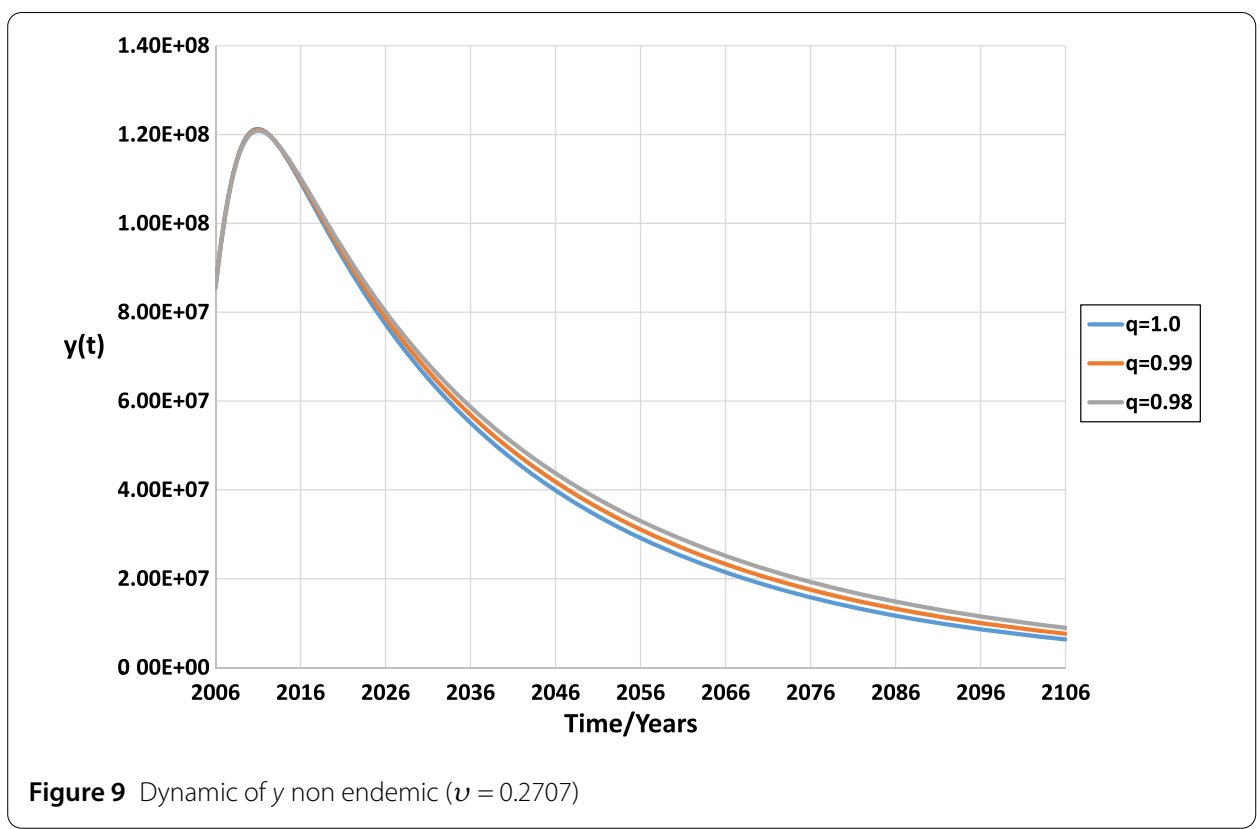

Consequently, we deduce that increasing the percentage $(v)$ of immunized population plays an important role in controlling the HBV spread. Additionally, in the fractional-order case, the peak of the infected individuals $y(t)$ is lower, which gives more accurate results resembling real data.

\section{Conclusions}

In this paper, the epidemic dynamics of fractional SIRS model on homogenous networks is studied.

We show that when $R_{0}<1$, the disease-free equilibrium point $E_{0}$ is locally and globally asymptotically stable, namely, the extinction of the disease will be achieved. When $R_{0}>1$, the endemic equilibrium point $E_{1}$ is also locally and globally asymptotically stable, mean- 

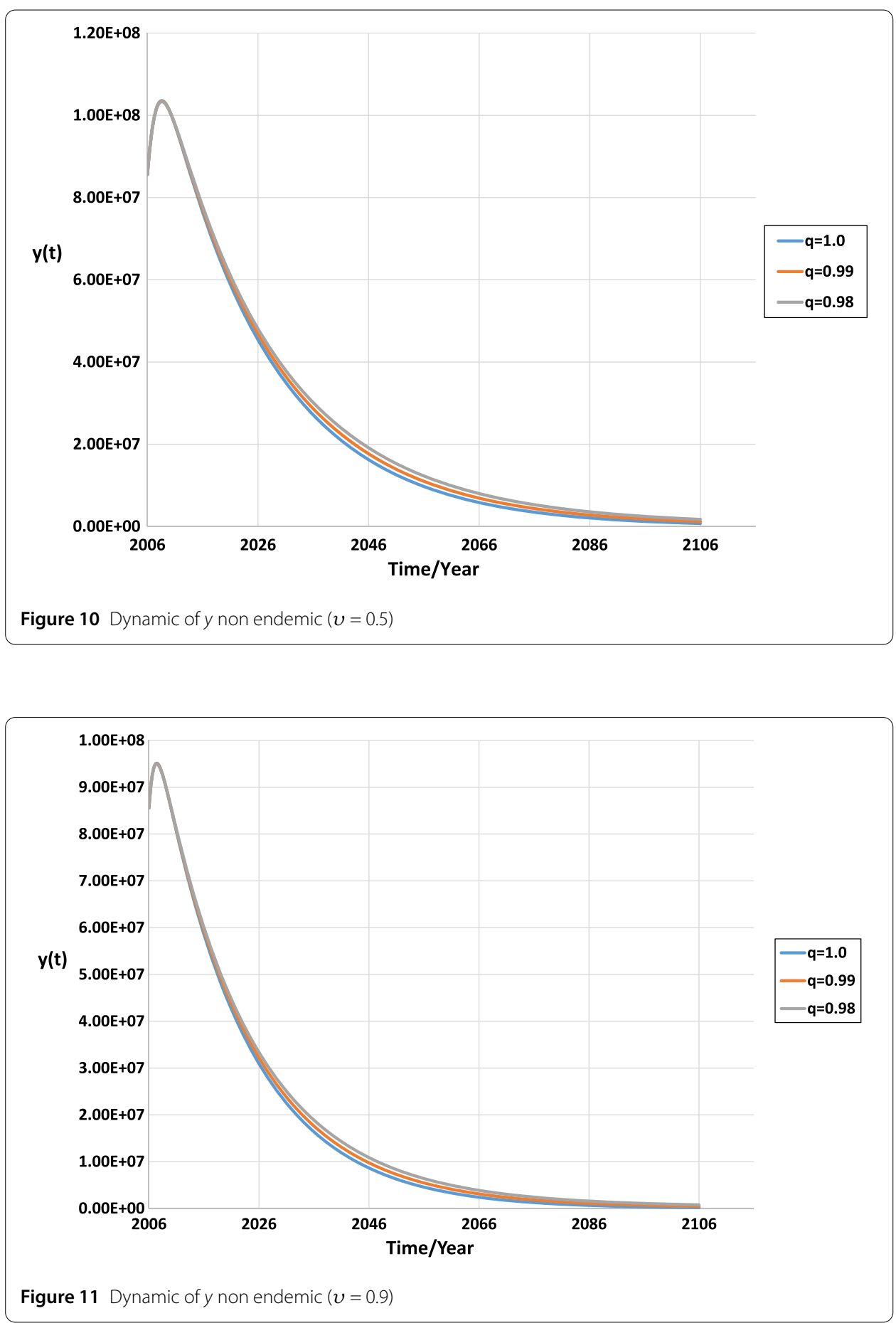

ing that the disease will persist in the population. The theoretical analysis is supported by numerical simulations. Numerical simulations show that the fractional-order case has lower peak of the infection, and the obtained results better resemble real data.

We notice that the fractional mathematical modeling is in many cases a more powerful and accurate approach to epidemiological models, based on the better suitability to fractional order that best corresponds to real data. 
Table $3(v=0.2707)$

\begin{tabular}{ll}
\hline$q$ & Year \\
\hline 1.0 & 2098 \\
0.99 & 2103 \\
0.98 & 2110 \\
\hline
\end{tabular}

Table $4(v=0.5)$

\begin{tabular}{ll}
\hline$a$ & Year \\
\hline 1.0 & 2061 \\
0.99 & 2064 \\
0.98 & 2068 \\
\hline
\end{tabular}

Table $5(v=0.9)$

\begin{tabular}{ll}
\hline$q$ & Year \\
\hline 1.0 & 2049 \\
0.99 & 2051 \\
0.98 & 2054 \\
\hline
\end{tabular}

\section{Acknowledgements}

We thank Prof. E. Ahmed (Mathematics Department, Faculty of Science, Mansoura University, Mansoura, Egypt) for his support and comments.

Funding

Not applicable.

\section{Competing interests}

The authors declare that they have no competing interests.

\section{Authors' contributions}

All authors contributed equally to the writing of this paper. They read and approved the final version of the manuscript.

\section{Author details}

${ }^{1}$ Mathematics Department, Faculty of Science, Damietta University, New Damietta, Egypt. ${ }^{2}$ Mathematics \& Computer science Department, Faculty of Science, Port Said University, Port Said, Egypt.

\section{Publisher's Note}

Springer Nature remains neutral with regard to jurisdictional claims in published maps and institutional affiliations.

\section{Received: 22 August 2018 Accepted: 3 April 2019 Published online: 18 April 2019}

\section{References}

1. Dorogovtsev, S.N., Goltsev, A.V., Mendes, J.F.F.: Critical phenomena in complex networks. Rev. Mod. Phys. 80(4), $1275-1335(2008)$

2. Xu, D., Xu, X., Yang, C., Gui, W.: Global stability of a variation epidemic spreading model on complex networks. Math. Probl. Eng. 2015, Article ID 365049 (2015)

3. Newman, M.E.J.: The structure and function of complex networks. SIAM Rev. 45(2), 167-256 (2003)

4. Kephart, J.O., White, S.R., Chess, D.M.: Computers and epidemiology. IEEE Spectr. 30(5), $20-26$ (1993)

5. Hu, Y., Mina, L., Kuang, Y.: Modeling the dynamics of epidemic spreading on homogenous and heterogeneous networks. Appl. Anal. 94(11), 2308-2330 (2014)

6. Li, H., Zhang, L., Hu, C., Jiang, Y., Teng, Z.: Dynamic analysis of a fractional order single-species model with diffusion. Nonlinear Anal., Model. Control 22(3), 303-316 (2017)

7. Li, H., Ahmadjan, M., Zhang, L., Teng, Z:: Stability analysis of a fractional-order predator prey model incorporating a constant prey refuge and feedback control. Adv. Differ. Equ. 2018, 325 (2018)

8. El-Saka, H.A.A., Ahmed, E.: A fractional order network model for ZIKA. https://doi.org/10.1101/039917 (2016) First posted 18 Feb 2016

9. Podlubny, l.: Fractional Differential Equations. Academic Press, San Diego (1999)

10. Ahmed, E., El-Sayed, A.M.A., El-Saka, H.A.A.: On some Routh-Hurwitz conditions for fractional order differential equations and their applications in Lorenz, Rössler, Chua and Chen systems. Phys. Lett. A 358, 1-4 (2006)

11. Ahmed, E., El-Sayed, A.M.A., El-Saka, H.A.A.: Equilibrium points, stability and solutions of fractional-order predator-prey and rabies models. J. Math. Anal. Appl. 325, 542-553 (2007)

12. El-Saka, H.A., El-Sayed, A.: Fractional Order Equations and Dynamical Systems. Lambert Academic Publishing, Germany (2013). ISBN 978-3-659-40197-8 
13. Matignon, D.: Stability results for fractional differential equations with applications to control processing. In: Computational Eng. in Sys. Appl., Lille France, vol. 2, pp. 963-968 (1996)

14. Huo, J., Zhao, H., Zhu, L.: The effect of vaccines on backward bifurcation in a fractional order HIV model. Nonlinear Anal., Real World Appl. 26, 289-305 (2015)

15. Vargas-De-León, C.: Volterra-type Lyapunov functions for fractional-order epidemic systems. Commun. Nonlinear Sci. Numer. Simul. 24(1), 75-85 (2015)

16. Diethelm, K.: The Analysis of Fractional Differential Equations: An Application-Oriented Exposition Using Differential Operators of Caputo Type. Lecture Notes in Mathematics. Springer, Berlin Heidelberg (2010)

17. El-Mesiry, E.M., El-Sayed, A.M.A., El-Saka, H.A.A.: Numerical methods for multi-term fractional (arbitrary) orders differential equations. Appl. Math. Comput. 160(3), 683-699 (2005)

18. El-Sayed, A.M.A., El-Mesiry, E.M., El-Saka, H.A.A.: Numerical solution for multi-term fractional (arbitrary) orders differential equations. Comput. Appl. Math. 23(1), 33-54 (2004)

19. The Jakarta Post: Healthcare stumbling in Rl's hepatitis fight.

http://www.thejakartapost.com/news/2011/01/13/healthcare-stumbling-ri\%E2\%80\%99s-hepatitis-fight.html (2011)

20. Wikipedia: Hepatitis B. http://en.wikipedia.org/wiki/Hepatitis_B (2013)

\section{Submit your manuscript to a SpringerOpen ${ }^{\circ}$ journal and benefit from:}

- Convenient online submission

Rigorous peer review

- Open access: articles freely available online

- High visibility within the field

- Retaining the copyright to your article

Submit your next manuscript at $\gg$ springeropen.com 